\title{
0 ensino baseado em projeto e a aprendizagem colaborativa com sistemas de informação geográfica
}

\author{
Tatiana Silva da Silva \\ Departamento de Geodésia, Instituto de Geociências, \\ UFRGS, Porto Alegre - RS. \\ tatiana.silva@ufrgs.br \\ Flávia Cristiane Farina \\ Departamento de Geodésia, Instituto de Geociências, \\ UFRGS, Porto Alegre - RS. \\ flavia.farina@ufrgs.br \\ Jorge Luiz Barbosa da Silva \\ Departamento de Geodésia, Instituto de Geociências, \\ UFRGS, Porto Alegre - RS. \\ jorge.silva@ufrgs.br \\ Ricardo Norberto Ayup-Zouain \\ Departamento de Geodésia, Instituto de Geociências, \\ UFRGS, Porto Alegre - RS. \\ ricardo.ayup@ufrgs.br
}

\begin{abstract}
PROBLEM BASED LEARNING AND COLLABORATIVE LEARNING WITH GEOGRAPHIC INFORMATION SYSTEMS. Geographic Information Systems (GIS) are a branch of technology applicable to virtually all knowledge areas. Traditional GIS teaching is normally based on lectures and tutorials, which does not prepare the student to use the tool in the intercommunication among professionals and in real problem solving. This article aims to determine the impact of problem solving-based GIS teaching on the skills acquired by the GIS students. To achieve it, the course was divided in two phases; the first was based on lectures and tutorials, and the second one based on problem solving. A poll was carried out at the end of each phase through the NAVi platform questioning students on how able they feel to use GIS professionally. More than $60 \%$ of the students showed a performance improvement. The results show the importance and effectiveness of problem-based collaborative learning in GIS teaching.
\end{abstract}

Citation: Silva T. S.da., Farina F.C., Silva J.L.B.da., Ayup-Zouain R. N. 2013. 0 ensino baseado em projeto e a aprendizagem colaborativa com sistemas de informação geográfica. Terræ Didatica, 9(1):14-21. <http://www.ige.unicamp.br/terraedidatica/>.

KEYWORDS: Geology, teaching using projects, cooperative learning, geographical information systems.

RESUMO Sistemas de Informação Geográfica (SIG) constituem uma tecnologia aplicável a praticamente todas as áreas de conhecimento. O ensino tradicional em SIG normalmente é baseado em aulas teóricas e exercícios tutoriais, o que não prepara o aluno para seu uso na intercomunicação entre profissionais e solução de problemas reais. O objetivo deste trabalho é definir o impacto do ensino de SIG baseado na solução de problemas sobre as habilidades adquiridas pelos alunos de SIG. Para tal, a disciplina foi dividida em duas fases, sendo a primeira focada em teoria e exercícios tutoriais e, a segunda, na solução de problemas. Ao final de cada fase foi feita uma enquete na plataforma NAVi sobre quão apto o aluno se sente para usar um SIG profissionalmente. Mais de $60 \%$ dos alunos responderam ter uma melhora no desempenho. Os resultados demonstram a importância e eficácia da aprendizagem colaborativa baseada em projeto no ensino de SIG.

PALAVRAS-CHAVE: Geologia, ensino por projeto, aprendizagem colaborativa, sistemas de informação geográfica. 


\section{Introdução}

Segundo Burrough \& McDonnel (1998), os Sistemas de Informação Geográfica (SIG) são um conjunto poderoso de ferramentas para a coleta, estocagem, recuperação, transformação e visualização de dados espaciais do mundo real para um conjunto particular de objetivos. Esses sistemas computadorizados sobrepõem diversos tipos de informação espacialmente explícita sobre uma área. A informação é apresentada visualmente ou graficamente, estando disponível de forma eficiente para comparações e correlações (Forman 1995). Como virtualmente todos os processos naturais e humanos dependem direta ou indiretamente do espaço, os SIGs podem ser aplicados na busca de soluções nas mais diversas áreas de conhecimento. São de extrema importância nas arenas da tomada de decisão e formulação de políticas. Além disso, normalmente envolvem uma visão holística sobre os problemas com que lidam, uma vez que a forma que os processos se distribuem ao longo do espaço na superfície da Terra depende de uma série de fatores, sejam eles físicos, químicos, biológicos ou humanos. Uma abordagem interdisciplinar é inevitável. Assim, o SIG naturalmente se apresenta como um sistema que promove e facilita atividades colaborativas com o suporte computacional, promovendo a comunicação entre diversas disciplinas.

Desde 1994 educadores em geografia e outros professores pioneiros têm integrado o SIG a várias aplicações curriculares (Alibrandi \& Baker 2008). Em menos de duas décadas o ensino de SIG migrou de poucos cursos específicos em poucos departamentos acadêmicos para se tornar um dos elementos principais de quase todos os programas de ensino em geografia e meio ambiente, com uma presença crescente em outras disciplinas também (DiBiasi et al. 2011). Na qualidade de uma tecnologia e disciplina muito baseada no intelecto, simplesmente mostrar mecanicamente como usar um programa computacional de SIG pela aplicação de de rotinas prontas configura uma postura antiética. A fim de gerar a capacidade de dominar esta tecnologia, o objetivo final do ensino em SIG deveria estar focado no seu uso como uma extensão do pensamento analítico do aluno, e não como uma "caixa preta". Com base nesta premissa, novas abordagens vêm sendo buscadas para superar as limitações que o uso exclusivo de exercícios tutoriais acarreta e, assim, não somente ensinar sobre o SIG, mas com o SIG.
Portanto, o ensino tradicional em SIG não faz uso de todas suas potencialidades. Normalmente ele é baseado em aulas teóricas combinadas a exercícios tutoriais. Esta prática introduz os conceitos e procedimentos técnicos básicos envolvidos na manipulação de dados espaciais, mas não capacitam verdadeiramente o aluno a lidar com problemas reais, complexos e multideterminados, a serem tratados por equipes multidisciplinares, o que constituirá sua realidade futura na vida profissional. De acordo com modelo da pedagogia relacional (Becker 1999), a educação inclui pesquisa, investigação e solução de problemas, não somente a transmissão de conhecimento, demonstração e aplicação de modelos (Silva et al. 2007).

O Departamento de Geodésia da Universidade Federal do Rio Grande do Sul é um dos tantos a atualmente oferecer a disciplina de SIG. Esta disciplina reflete de certa forma a diversidade de situações encontrada nas aplicações reais de SIG. As turmas são compostas por alunos de início de curso a formandos, com diferentes formações em termos curriculares (Geologia, Engenharia Civil, Engenharia Cartográfica e Engenharia Ambiental), e diferentes visões sobre questões como meio ambiente, planejamento e gestão. Além disso, as aplicações previstas no currículo incluem fenômenos de escala local à global. Tais disparidades não parecem ser uma exclusividade local, mas uma característica comum no ensino em SIG ao redor do mundo, como evidenciado na sessão "Teaching in Land Change Science" da Global Land Project Open Science Meeting 2010 (GLP 2010).

O que inicialmente soa como uma dificuldade para o ensino, na verdade constitui um cenário riquíssimo para o desenvolvimento de habilidades, o que não seria possível em turmas homogêneas em termos de formação e visão de mundo. Tais condições permitem avaliar como os alunos trabalham em conjunto a fim de encontrar soluções para problemas que envolvem a interdisciplinariedade e como a tecnologia SIG pode ser usada para facilitar este processo.

Segundo Kopp \& Mandl (2002), o ensino tradicional assume que o conhecimento é uma consequência do aprendizado de fatos e rotinas e pode ser transmitido de uma pessoa para outra como produtos. Considera-se que o aluno pode fazer as ligações entra a teoria e a prática por si só. Já na visão construtivista, segundo os mesmos autores, o processo de ensino-aprendizagem apresenta seis 
características essenciais: (1) a aprendizagem é um processo motivacional: os alunos têm que querer aprender alguma coisa e, frequentemente, o esforço de aprendizagem é determinado pela motivação e interesse do aluno sobre o tópico que ele deve aprender; (2) a aprendizagem é um processo ativo: o aprendizado efetivo é somente possível quando o aluno é ativo, sendo a motivação e interesse antecedentes necessários para a atividade; (3) a aprendizagem é um processo auto-regulado: o aluno deve ter a chance de controlar seu próprio processo de aprendizagem enquanto lida com certo conteúdo; (4) a aprendizagem é um processo construtivista: o aluno somente pode adquirir e usar o conhecimento quando pode integrar o novo conhecimento ao antigo por meio das estruturas já existentes em sua mente e quando ele pode interpretá-lo com base na sua experiência individual; (5) a aprendizagem é um processo de situação: o conhecimento está sempre relacionado a alguma situação, de forma que a aquisição de conhecimento está conectada a um contexto específico; e (6) a aprendizagem é um processo social: a aquisição de conhecimento normalmente toma parte na interação com outros, sendo a aprendizagem um processo que pertence a uma cultura específica de aprendizado, na qual os conteúdos, bem como os valores e atitudes, são negociados.

$\mathrm{Na}$ realização dos projetos os alunos devem vencer desafios, realizar investigações e pesquisas e tudo isto tem caráter motivador, centrando o foco do processo de ensino-aprendizagem no aluno, o qual realiza uma aprendizagem ativa (Teixeira et al. 2006). As características inerentes do trabalho com SIG são o palco perfeito para tal, proporcionando aos alunos a própria construção do conhecimento. Segundo a metodologia da problematização, os problemas são identificados pelos alunos por meio da observação da realidade, onde as questões de estudo estão acontecendo, e assim formulam hipóteses de solução, que orientarão a intervenção na realidade da qual se extraiu o problema. O grupo trabalha junto o tempo todo, com a supervisão do professor. Em síntese, trata-se de uma oportunidade de aprendizagem efetiva, no contato e no confronto o mais direto possível com a realidade, onde a ação humana ou os fenômenos da natureza ocorrem concretamente (Berbel 1998).

As metodologias problematizadoras surgiram no pós-guerra, como uma das estratégias de reconstrução dos países fortemente destruídos. Mas é na década de 70 que essas metodologias aparecem nos países latino-americanos como forma de melhorar o padrão de ensino-aprendizagem nos cursos de graduação (Farah \& Pierantoni 2003). No ensino em SIG, a adoção de uma abordagem baseada na solução de problemas tem permitido aos estudantes integrar questionamentos, conceitos e métodos ao se deparar com situações do mundo real (Drennon 2005).

No cenário atual, onde os recursos naturais sofrem uma crescente pressão das atividades humanas, deles dependentes, medir e manejar tais recursos se tornou uma tarefa essencial a fim de sustentar a vida humana no planeta. Uma das principais vantagens do SIG é justamente permitir uma melhor gestão de recursos. Sua característica de usar a localização geográfica para associar diferentes dados ambientais permite solucionar problemas de alocação bem como diversos outros relacionados ao processo de tomada de decisão e de definição de estratégias de manejo. Além disso, a ligação entre bancos de dados por meio de dados locacionais em comum permite o compartilhamento de informação entre instituições públicas e privadas. Recursos humanos capacitados a lidar com este tipo de tecnologia constituem uma demanda atual nas mais diversas organizações e aplicações. Esta necessidade por pessoas com domínio sobre as geotecnologias tende a aumentar devido à crescente busca da sociedade por soluções em planejamento urbano e ambiental. Somente esta justificativa deveria ser um incentivo à educação superior para investir no ensino de SIG em nível de graduação (Attard 2008). Mas ainda existe uma clara lacuna de estratégias de ensino e aprendizado para o uso de tecnologias na sala de aula (Kinniburgh 2010).

As universidades da União Europeia atualmente contam com um número significativo de professores de SIG com grande experiência e, globalmente, se prevê uma importante competência vinda dos países terceiro mundistas (Melero 2005). Segundo Fuling \& Shaohua (2008), a universidade deve se tornar a força principal no desenvolvimento da indústria SIG, sendo o Estado o responsável por promulgar políticas relevantes para vigorosamente desenvolver o ensino superior em SIG. Assim, ao ensino formal e não-formal em SIG cabe a responsabilidade de gerar a mão-de-obra qualificada a fim de tentar suprir tais demandas da sociedade atual brasileira.

Neste contexto, o objetivo do presente trabalho é avaliar o impacto da abordagem baseada 
em projeto no ensino de sistemas de informação geográfica (SIG). Para tal, dois tipos de abordagens de ensino foram comparados na disciplina Sistema Geográfico de Informações oferecida pelo Departamento de Geodésia da UFRGS. O primeiro é baseado em aulas teóricas expositivas e exercícios tutoriais e foi aplicado nos primeiros dois meses da disciplina. O segundo é baseado na solução de problemas com base em SIG por grupos de alunos provenientes de diferentes cursos de graduação, o que foi realizado nos dois meses finais da disciplina.

\section{Materiais e métodos}

A fim de determinar o impacto do ensino baseado em projeto, tendo o SIG como ferramenta de aprendizagem colaborativa na solução de problemas, a disciplina de Sistemas Geográficos de Informação oferecida pelo Departamento de Geodésia da UFRGS foi dividida em duas fases, como dito anteriormente. Na primeira parte da disciplina somente os conceitos teóricos foram introduzidos, acompanhados de exercícios tutoriais, com foco no domínio dos procedimentos básicos em SIG. Apesar de abertas discussões iniciais e finais sobre os problemas propostos pelos tutoriais e as soluções encontradas, o desenvolvimento dos exercícios foi feito em base individual.

$\mathrm{Na}$ segunda parte da disciplina, os alunos foram convidados a formar equipes multidisciplinares (grupos de colaboração formados por alunos de diferentes cursos) que deveriam encontrar soluções para problemas reais, com base em SIG, para áreas e temas de livre escolha. Esta fase segue a metodologia da problematização, onde os alunos identificam um problema real e planificam soluções a serem tratadas dentro do escopo da disciplina.

A estrutura de pequenas equipes multidisciplinares é uma característica real das atividades profissionais envolvendo o uso de SIG (ver seção anterior), transformando em uma vantagem a diversidade dos estudantes da disciplina. Os grupos são aconselhados a escolherem áreas geográficas próximas, permitindo a visualização in situ dos processos discutidos em aula. Todas as etapas de trabalho são definidas pelos grupos de alunos, com o suporte do professor, desde a obtenção de dados, definição de procedimentos metodológicos a serem adotados, até a escolha da solução ou conjunto de alternativas de solução apropriadas ao problema em questão.
Ao final de cada fase da disciplina foi feita uma enquete por meio da plataforma NAVi. O NAVi constitui um ambiente de educação a distância (EAD) desenvolvido com o intuito de apoiar o processo de aprendizagem na UFRGS, nas áreas de ensino (nos níveis de graduação de pós-graduação, nas modalidades presencial e a distância), pesquisa e extensão. Diferencia-se essencialmente pelas características de ênfase na interação, enfoque sistêmico, uso de vídeos e videochats, simplicidade de navegação e autonomia de atualização pelos professores (NAVi 2011). Por meio da ferramenta "Enquete" foi perguntado ao aluno quão apto ele se consideraria, caso fosse contratado para atuar dentro de uma equipe multidisciplinar em projetos auxiliados por SIG. Quatro alternativas de resposta foram apresentadas:

(a) apto a construir e executar projetos auxiliados por SIG;

(b) apto a discutir como o SIG poderia ser aplicado na busca de soluções;

(c) apto a ressaltar que o SIG poderia auxiliar, mas não saberia explicar exatamente como; e

(d) nenhuma das anteriores.

As enquetes foram aplicadas em quatro turmas entre 2011 e 2012, totalizando 44 alunos. Os projetos desenvolvidos pelos alunos incluíram principalmente as seguintes aplicações, embora não estejam restritos a elas:

- geomarketing;

- multimídias cartográficas;

- alocação de infraestruturas;

- sistema de apoio à gestão municipal;

- modelos demográficos;

- cenários de valorização imobiliária frente à Copa;

- monitoramento da silvicultura; e

- avaliação comparativa de fontes de dados topográficos.

As aplicações de SIG de uma maneira geral podem ser classificadas, em ordem de complexidade, nos seguintes grupos: mapeamento de recursos, construção de bancos de dados geográficos e modelagem espacial (Eastman 2009). Cabe ressaltar que a maioria dos temas desenvolvidos pelos alunos em seus projetos envolveu em maior ou menor grau procedimentos de modelagem espacial, o que requer uma elevada capacidade de abstração e entendimento da representação de dados geográficos em meio digital e dos procedimentos de manipulação dos mesmos. 


\section{Resultados e discussão}

As figuras 1, 2, 3 e 4 apresentam os resultados da enquete, respectivamente, para as turmas A e B, do primeiro semestre de 2011, U do segundo semestre de 2011 e U do segundo semestre de 2012. Todas as turmas, ao final da primeira fase da disciplina, baseada em aulas teóricas e exercícios tutoriais, optaram em maioria pela resposta (b), ou seja, aptos a discutir como o SIG poderia ser aplicado na busca de soluções, mas não a construir

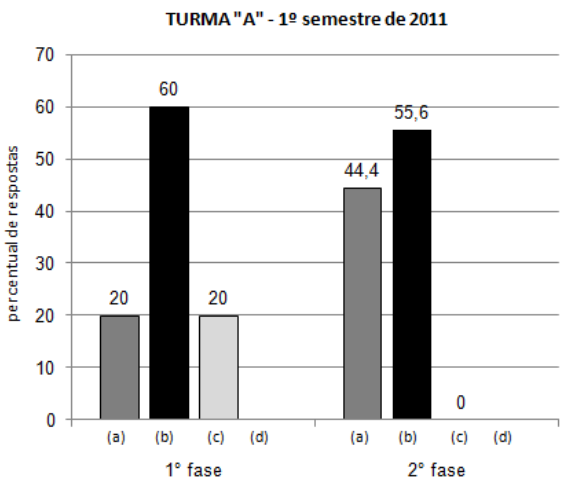

Figura 1. Resultados da enquete feita com a Turma "A" de 2011 sobre o nível de aptidão dos alunos ao uso profissional de SIG antes ( $1^{\circ}$ fase) e depois ( $2^{\circ}$ fase) do desenvolvimento de projetos. Respostas: (a) apto a construir e executar projetos auxiliados por SIG; (b) apto a discutir como o SIG poderia ser aplicado na busca de soluções; (c) apto a ressaltar que o SIG poderia auxiliar, mas não saberia explicar exatamente como; e (d) nenhuma das anteriores.

TURMA "U" - 20 semestre de 2011

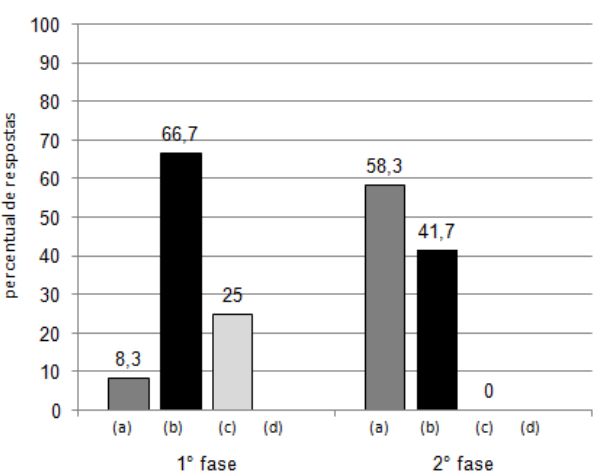

Figura 3. Resultados da enquete feita com a Turma "U" de 2011 sobre o nível de aptidão dos alunos ao uso profissional de SIG antes ( $1^{\circ}$ fase) e depois ( $2^{\circ}$ fase) do desenvolvimento de projetos. Respostas: (a) apto a construir e executar projetos auxiliados por SIG; (b) apto a discutir como o SIG poderia ser aplicado na busca de soluções; (c) apto a ressaltar que o SIG poderia auxiliar, mas não saberia explicar exatamente como; e (d) nenhuma das anteriores. e executar projetos auxiliados por SIG. O total de $60 \%$ dos alunos da turma A, 100\% dos alunos da turma B, 66,7\% da turma U/2011 e 71,4\% da turma U/2012 optaram por esta resposta. Do restante dos alunos da turma A, $20 \%$ já se consideravam aptos a construir e executar projetos auxiliados por SIG e outros $20 \%$ disseram estar aptos a ressaltar que o SIG poderia auxiliar, mas não saberia explicar exatamente como. As turmas U/2011 e U/2012 mostraram um padrão semelhante, sendo que do

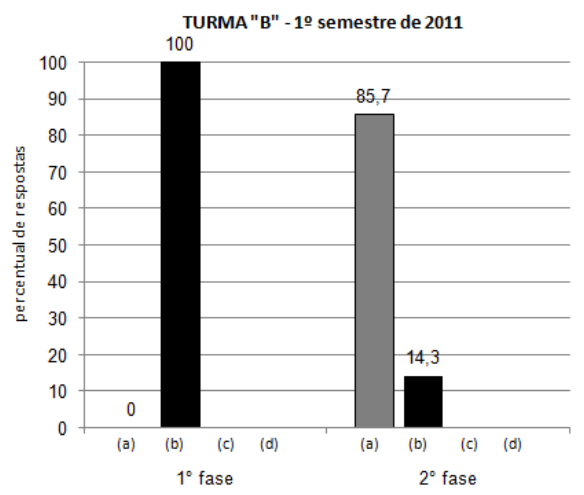

Figura 2. Resultados da enquete feita com a Turma "B" de 2011 sobre o nível de aptidão dos alunos ao uso profissional de SIG antes ( $1^{\circ}$ fase) e depois $\left(2^{\circ}\right.$ fase) do desenvolvimento de projetos. Respostas: (a) apto a construir e executar projetos auxiliados por SIG; (b) apto a discutir como o SIG poderia ser aplicado na busca de soluções; (c) apto a ressaltar que o SIG poderia auxiliar, mas não saberia explicar exatamente como; e (d) nenhuma das anteriores.

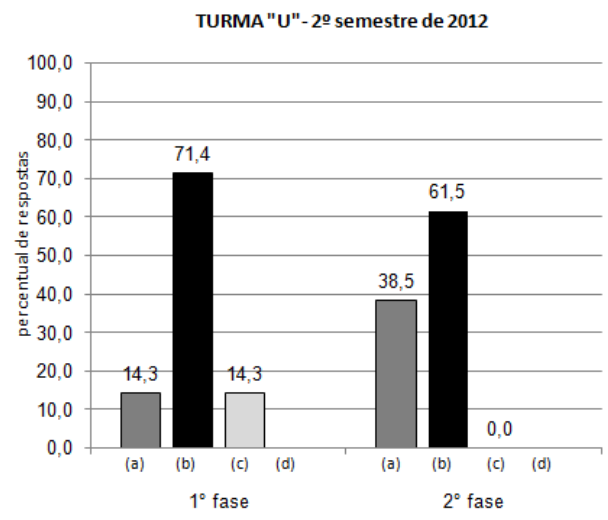

Figura 4. Resultados da enquete feita com a Turma "U" de 2012 sobre o nível de aptidão dos alunos ao uso profissional de SIG antes ( $1^{\circ}$ fase) e depois ( $2^{\circ}$ fase) do desenvolvimento de projetos. Respostas: (a) apto a construir e executar projetos auxiliados por SIG; (b) apto a discutir como o SIG poderia ser aplicado na busca de soluções; (c) apto a ressaltar que o SIG poderia auxiliar, mas não saberia explicar exatamente como; e (d) nenhuma das anteriores. 
restante de seus alunos, 8,3\% na turma U/2011 e $14,3 \%$ na turma U/2012 já se consideravam aptos a construir e executar projetos auxiliados por SIG e outros $25 \%$ e $14 \%$, para as turmas U de 2011 e 2012 respectivamente, disseram estar aptos a ressaltar que o SIG poderia auxiliar, mas não saberia explicar exatamente como.

Ao final da segunda fase da disciplina, após ter sido planejado e executado o projeto de solução de um problema real em SIG, 44,4\% dos alunos da turma A, $85,7 \%$ dos alunos da turma B, $58,3 \%$ da turma U/2011 e 38,5\% da turma U/2012 se consideraram aptos a construir e executar projetos auxiliados por SIG. O restante de todas as turmas optou pela resposta (b) e nenhum pela resposta (c), ou seja, todos os alunos se consideram aptos pelo menos a discutir como o SIG poderia ser aplicado na busca de soluções em sua vida profissional após a execução do projeto final da disciplina, senão a planejar e executar projetos auxiliados por SIG.

Nota-se que, mesmo ao final da $1^{\circ}$ fase da disciplina, somente baseada em aulas teóricas e exercícios tutoriais, todos os alunos se consideraram aptos pelo menos a ressaltar em que situações o uso de SIG poderia ser aplicado na solução de problemas, embora nem todos pudessem explicar em detalhes quais procedimentos seriam mais adequados a estas aplicações neste momento. Entretanto, ao final da $2^{\circ}$ fase da disciplina, quando é inserida a abordagem baseada em projeto, todos os alunos passaram a se considerar aptos não somente a apontar as situações em que o SIG poderia ser aplicado, mas também a justificar sua posição. Destes, mais de 50\% disseram estar aptos a construir e executar projetos auxiliados por SIG, um resultado superior ao esperado, segundo as metas definidas para a disciplina.

Segundo Reinmann-Rothmeier \& Mandl (2001), para planejar ambientes de aprendizagem baseados em problema, quatro princípios devem ser considerados:

(1) Autenticidade e referência à aplicação: um resumo do conhecimento deve ser apresentado usando exemplos práticos, problemas ou estudos de caso para mostrar sua relevância no campo profissional. Simultaneamente, esta forma de fornecer conteúdo mostra a aplicabilidade do conhecimento e aumenta a motivação e interesse dos alunos em um tópico específico.

(2) Contextos e perspectivas múltiplas: para evitar o conhecimento engessado a certo contexto, os tópicos devem ser apresentados em situações diferentes e sob diferentes perspectivas. Esta forma de mostrar múltiplos contextos e perspectivas evoca um conhecimento mais flexível sobre um assunto. Ao mesmo tempo, este método estimula a habilidade de analisar coisas de formas diferenciadas e de aplicar o conhecimento em outras circunstâncias.

(3) Arranjos sociais de aprendizagem: embora a aprendizagem seja um processo individual, aspectos sociais se tornam cada vez mais importantes no processo de aquisição do conhecimento, pois a habilidade de se comunicar e expressar é essencial em todas as profissões. Além disso, trabalhar com tarefas em grupo é uma forma adequada de apoiar e aprofundar o entendimento dos alunos sobre os conteúdos e suas conexões.

(4) Instruções, informações e provisão da construção. As instruções são úteis aos alunos como apoio ao trabalhar em tarefas em um ambiente de aprendizagem. Assim, é importante que este ambiente não demande demais dos alunos a fim de evitar uma sobrecarga cognitiva. Isto, entretanto, não deve diminuir o compromisso. Ao contrário, com a ajuda de certas instruções, o aluno deve ser encorajado a pesquisar por si próprio os detalhes das informações que precisa para realizar suas tarefas.

A abordagem baseada em projeto, como apresentada neste trabalho, mostra características em comum com a abordagem baseada em problema e, consequentemente, compartilha pontos positivos. Embora o problema a ser solucionado pelos alunos na segunda fase da disciplina é eleito por eles, a primeira fase apresenta a teoria de SIG acompanhada de inúmeros estudos de caso e exemplos de aplicações. O interesse por temas e/ou aplicações específicas surge neste momento em função de seu histórico cognitivo e características individuais. Uma vez iniciada a segunda fase da disciplina, onde um grupo interdisciplinar é formado e o projeto é definido, diferentes visões sobre o mesmo problema podem surgir. O SIG se apresenta como uma ferramenta de intercomunicação, reunindo o conhecimento do grupo e gerando novas informações, possibilitando, por meio de mapas, a visualização de diferentes parâmetros, estruturas de decisão e soluções.

As investigações baseadas em SIG dão suporte à teoria de aprendizado construtivista, e quando combinadas com problemas autênticos e reais, permite o aprendizado efetivo do aluno (Kinniburgh 2010). Também segundo Kinniburgh (op.cit.), não é apropriado que os alunos sejam ensinados usando métodos tradicionais baseados na disseminação direta de conhecimento e fatos. Os alunos devem 
poder explorar seus tópicos com encorajamento e flexibilidade de forma que a experiência de aprendizado seja válida. Uma atividade sem fim pré-determinado permite que as interações multidimensionais sejam exploradas entre diferentes áreas do aprendizado ao mesmo tempo em que os estudantes são expostos a poderosas informações e tecnologias da comunicação na forma de um SIG.

Os resultados aqui apresentados constituem um exemplo da superioridade das estratégias baseadas em projeto, com o uso computacional de SIG na prática, quando comparadas às aulas tradicionais expositivas e baseadas em exercícios tutoriais, nas quais a contribuição dos ambientes digitais é limitada. Embora não tenha sido o foco de investigação neste momento, é esperado que também haja uma grande vantagem no aprendizado das disciplinas correlatas envolvidas na solução dos problemas (projetos) trabalhados. O poder de comunicação por meio de mapas apresentado pelo SIG e/ou a própria necessidade do aluno em compreender processos físicos e humanos para chegar às soluções procuradas deve ter repercussões positivas no entendimento de outras áreas da ciência.

Drennon (2005) aponta que um dos pontos principais na inovação da formação em SIG é dar a oportunidade aos estudantes de crescer com a prática e entende que o conhecimento genuíno vem da prática. Outro ponto é o treinamento do espírito de grupo, uma vez que o desenvolvimento da ciência moderna tem maior ênfase na sabedoria coletiva.

Nos moldes em que a disciplina de SIG do Departamento de Geodésia (UFRGS) vem sendo ministrada nos últimos anos, o SIG é o objeto de estudo ao mesmo tempo em que constitui o ambiente de aprendizagem. Considerando a abordagem de ensino adotada, ela poderia ser classificada como ensino baseado em projeto (ou ensino baseado na solução de problemas), onde o foco principal é a aprendizagem de SIG. Entretanto, também compartilha de características e vantagens do ensino baseado em problema, já que o conhecimento dos aspectos inerentes aos problemas tratados em diferentes áreas do conhecimento (da ecologia ao marketing) não são ensinados durante a disciplina, mas acaba por ser construído ao longo do desenvolvimento dos projetos.

\section{Conclusão}

Apesar das deficiências do ensino tradicional em SIG, os resultados obtidos mostram um bom nível de entendimento por parte do aluno das questões aplicadas de SIG mesmo quando somente aulas expositivas e exercícios tutoriais são a base da estratégia de ensino. Ao final da $1^{\circ}$ fase do trabalho, baseada em aulas teóricas e exercícios tutoriais, as respostas das enquetes mostram que a totalidade dos alunos pelo menos compreende em que situações da vida real o SIG poderia ser aplicado na solução de problemas. Mais que isso, cerca de $11 \%$ dos alunos já se considera, mesmo nesta fase, apto a construir e executar projetos baseados em SIG. Entretanto, não foi analisada a possibilidade de que estes tivessem algum tipo de conhecimento ou experiência anterior em SIG.

Ainda assim, a realização de projetos colaborativos subsidiados por SIG, seguindo a metodologia da problematização, mostrou uma melhora significativa na capacidade dos alunos em se utilizar das ferramentas trabalhadas na solução de problemas reais. Os temas eleitos por eles, nas mais diferentes áreas de aplicação, representam problemas ou demandas reais e importantes no contexto atual e dos locais adotados como área de estudo, alguns deles com um alto nível de complexidade, não somente com relação ao entendimento do problema em si, que muitas vezes envolveram diversas dimensões, mas também no que concerne aos procedimentos técnicos requeridos para sua solução.

O ambiente SIG, pela sua característica inerente de requerer uma abordagem múltipla para a compreensão dos processos dependentes do espaço, em conjunto com as ferramentas oferecidas pela plataforma NAVi, permitiram e potencializaram as discussões em grupo. Desta forma, os alunos puderam observar diferentes parâmetros espaciais da área de estudo adotada, levantaram hipóteses sobre o conjunto de drivers principais envolvidos no problema trabalhado, determinaram os dados e procedimentos metodológicos necessários para a sua investigação e, por fim, chegaram a uma solução ou conjunto de alternativas de acordo com a temática de seus projetos. De uma maneira geral, os projetos superaram em muito o nível mínimo de dificuldade desejado para uma boa compreensão da disciplina.

Assim, os resultados obtidos mostram a importância e a eficácia da abordagem baseada em projeto e da aprendizagem colaborativa subsidiada por SIG, dada a melhora substancial no domínio das ferramentas expressa pelos alunos por meio das enquetes. Adicionalmente, a plataforma NAVi foi de grande valia não somente na interação entre alu- 
nos e professores para a execução dos projetos, mas também na avaliação de seus resultados, trazendo importantes subsídios para a adaptação da disciplina em questão, o que pode vir a ser replicado em outras áreas ou disciplinas.

\section{Referências bibliográficas}

Alibrandi M., Baker T. 2008. A social history of GIS in education, 1985-2007. In: Milson, A.J., Alibrandi, M. eds 2008. Digital Geography: Geospatial Technologies in the Social Studies Classroom. USA: Information Age Publishing. p. 3-37.

Attard M. Applying problem-based-learning to teach GIS in higher education. In: ESRI Intenternational User Conference, 2008, San Diego, Anais. URL: http://events.esri.com/uc/2008/proceedingsCD/educ/papers/pap_1363.pdf. Acesso: 18.04.2013.

Becker F. 1999. Modelos pedagógicos e modelos epistemológicos. Educação e Realidade. 19(1): 89-96.

Berbel N.N. 1998. A problematização e a aprendizagem baseada em problemas: diferentes termos ou diferentes caminhos? Interface - Comunicação, Saúde, Educação. 2(2):140-154.

Burrough P.A., McDonnell R.A. 1998. Principles of Geographic Information Systems. 2 ed. New York: Oxford. 352p.

DiBiasi D., Harvey F., Goranson C., Wright, D. 2011. The GIS Professional Ethics Project: pratical ethics for GIS professionals. In: Unwin, D., Tate, N. eds. 2011. Teaching Geographic Information Science and technology in higher education. West Sussex: Wiley-Blackwell. p. 199-210.

Drennon C. 2005. Teaching Geographic Information Systems in a problem-based learning environment. Journal of Geography in Higher Education. 29(3):385-402.

Eastman J.R. 2009. IDRISI Guide to GIS and Image Processing. Accessado em IDRISI Taiga. Worcester, MA: Clark Univ,: 342p.
Farah B.F., Pierantoni, C.R., 2003. A utilização da metodologia da Problematização no Curso Introdutório para Saúde da Família do Pólo de Capacitação da UFJF. Revista APS. 6(2):108-119.

Forman R.T.T. 1995. Land mosaics: the ecology of landscapes and regions. Grã-Bretanha: Cambridge University Press. 632p.

Fuling B., Shaohua, W. 2008.Problem, innovation and development of GIS higher education in our country. The International Archives of the Photogrammetry, Remote Sensing and Spatial Information Sciences. Beijing. Vol. 36. Part B6a.

GLP. 2010. Teaching in Land Change Science. Global Land Project Open Science Meeting 2010. URL: http://www.glp2010.org/E9.pdf. Acesso: 18.04.2013.

Kinniburgh J. 2010. A constructivist approach to using GIS in the New Zealand classroom. New Zealand Geographer. 66:74-84.

Kopp B., Mandl H. 2002. Problem-based learning in virtual GIS learning-environments. Third European GIS Education Seminar EUGISES. Girona: Spain.

Melero M.C. 2005. Problem based learning for GIS training. ICA Comission Seminar. Proceedings. Madrid.

NAVi 2011. Plataforma NAVi. Núcleo de Aprendizagem Virtual. UFRGS. URL: < https://www.ead. ufrgs.br/navi>. Acesso: 18.04.2013.

Reinmann-Rothmeier, G., Mandl, H. 2001. Unterrichten und Lernumgebungen gestalten. In: A. Krapp \& B. Weidenmann eds 2001, Pädagogische Psychologie. Weinheim: PsychologieVerlagsUnion. p: 601-646.

Silva R.P., Silva T.L.K, Teixeira R.G. 2007. Learning Objects: an approach in engineering education in a cognitive perspective. International Conference On Engineering Education. Anais. Coimbra.

Teixeira F.G, Silva R.P., Silva T.L.K., Hoffmann A.T. 2006. Geometria descritiva: aprendizagem baseada em projetos. In: Congr. Bras. Educ. em Engenharia, COBENGE, 34, 2006. Anais... Passo Fundo: Univ. Passo Fundo. 